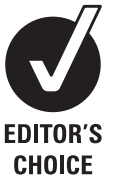

CHOICE
- Additional materials are published online only. To view these files please visit the journal online (http://dx.doi.org/ 10.1136/heartjnl-2012-302445).

${ }^{1}$ Department of Cardiology, 2nd Affiliated Hospital of Harbin Medical University, Key Laboratories of Education Ministry for Myocardial Ischemia Mechanism and Treatment, Harbin, China ${ }^{2}$ Cardiology Division, Massachusetts General Hospital, Harvard Medical School, Boston, Massachusetts, USA

${ }^{3}$ Biostatistics Center, Massachusetts General Hospital, Harvard Medical School, Boston, Massachusetts, USA

${ }^{4}$ Clinical Affairs, LightLab Imaging/St. Jude Medical, Westford, Massachusetts, USA

\section{Correspondence to}

Dr Bo Yu, Department of Cardiology, 2nd Affiliated Hospital of Harbin Medical University, Key Laboratories of Education Ministry for

Myocardial Ischemia Mechanism and Treatment, Harbin 150086, P.R. China; yubodr@163.com

Accepted 12 July 2012 Published Online First 6 August 2012

\title{
Significance of intraplaque neovascularisation for vulnerability: optical coherence tomography study
}

\author{
Jinwei Tian, ${ }^{1}$ Jingbo Hou, ${ }^{1}$ Lei Xing, ${ }^{1}$ Soo-Joong Kim, ${ }^{2}$ Taishi Yonetsu, ${ }^{2}$ Koji Kato, ${ }^{2}$ \\ Hang Lee, ${ }^{3}$ Shaosong Zhang, ${ }^{4}$ Bo Yu, ${ }^{1}$ Ik-Kyung Jang ${ }^{2}$
}

\begin{abstract}
Objectives This study aimed to investigate the role of intraplaque neovascularisation (NV) in culprit lesions and non-culprit lesions of unstable angina pectoris (UAP) and in lesions of stable angina pectoris (SAP) using optical coherence tomography (OCT).

Design This study was a retrospective study.

Setting The significance of NV for culprit and non-culprit plaques remains unclear.
\end{abstract}

Participants A total of 356 plaques from 92 UAP patients and 25 SAP patients who underwent OCT imaging were divided into three groups: culprit lesions in UAP (92), non-culprit lesions in UAP (203) and lesions of SAP (61).

Main outcome measures NV and plaque characteristics were examined by OCT and plaques with and without NV were compared.

Results Among UAP culprit lesions, plaques with NV had significantly higher incidence of thin cap fibroatheroma ( $81 \%$ vs $47 \%, p=0.002$ ) compared with those without NV. In addition, the fibrous cap was thinner $(56 \pm 20 \mu \mathrm{m}$ vs $75 \pm 30 \mu \mathrm{m}, \mathrm{p}<0.001)$, lipid arc was greater $\left(254 \pm 66^{\circ}\right.$ vs $222 \pm 65^{\circ}$, $p=0.024)$ and lipid core length was longer $(13 \pm 5 \mathrm{~mm}$ vs $10 \pm 6 \mathrm{~mm}, p=0.007)$. No significant difference was observed between non-culprit lesions of UAP with and without NV, and between lesions of SAP with and without NV.

Conclusion In patients with UAP, the culprit plaques with NV had vulnerable features such as thinner fibrous cap, greater lipid arc, longer lipid core length and more frequent thin cap fibroatheroma compared with those without NV. In both non-culprit lesions of UAP patients and in lesions of SAP patients NV was not associated with vulnerable plaque characteristics.

\section{INTRODUCTION}

Intraplaque neovascularisation (NV) derives mainly from pre-existing vasa vasorum in the adventitia and rarely from the luminal surface. ${ }^{1} \mathrm{NV}$ has been recognised as an important process for the progression of atherosclerotic plaques ${ }^{2-4}$ and as a potential therapeutic target for cardiovascular disease. ${ }^{5}$ Microvessels are more prevalent, larger and more irregular in symptomatic carotid plaques than in asymptomatic plaques. ${ }^{6} 7$ However, due to lack of available techniques, in vivo data on coronary plaque NV has been limited.

Optical coherence tomography (OCT) is an intracoronary imaging modality with resolution 10 times higher than that of intravascular ultrasound
(IVUS). OCT is useful in evaluating plaque structure at a microscopic level, including fibrous cap thickness, macrophage infiltration and lipid content. $^{8}$ OCT has been shown to be able to visualise microvessels within coronary atherosclerotic plaques and neointima inside stents in vivo. ${ }^{8-11}$

The present study was designed to investigate the significance of intraplaque NV in both culprit lesions and non-culprit lesions of unstable angina pectoris (UAP) and in lesions of stable angina pectoris (SAP) using OCT. We hypothesised that $\mathrm{NV}$ in unstable plaque is associated with features consistent with plaque vulnerability.

\section{METHODS}

\section{Study sample}

Between May 2008 and April 2011, consecutive patients who underwent successful OCT imaging within 2 weeks of presentation at the 2 nd Affiliated Hospital of Harbin Medical University were included in the analysis. SAP was defined as chest pain on exertion without changes in frequency, duration, or intensity of symptoms for more than 4 weeks along with positive nuclear stress test results. UAP was characterised by a progressive crescendo pattern or angina at rest without elevated cardiac markers. Patients were excluded when they had (1) left main disease; (2) chronic total occlusion; (3) an extremely tortuous or heavily calcified vessel; (4) congestive heart failure with left ventricular ejection fraction greater than $40 \%$; or (5) renal insufficiency with serum creatinine greater than $1.8 \mathrm{mg} / \mathrm{dl}$. Culprit lesions were identified by a combination of ECG changes, left ventricular wall motion abnormalities and angiographic lesion morphology. OCT examinations were performed in all patients, while IVUS examinations were performed only in 35 UAP patients (including 35 culprit lesions and 52 non-culprit lesions). Two cardiologists independently reviewed all clinical and angiographic data to decide angina status and the culprit lesions. A total of 356 plaques from 117 patients (92 UAP patients and 25 SAP patients) were divided into three groups: culprit lesions in UAP (92 patients, 92 plaques), nonculprit lesions in UAP (92 patients, 203 plaques) and lesions in SAP (25 patients, 61 plaques).

This study was approved by the ethics committee of the 2nd Affiliated Hospital of Harbin Medical University (Harbin, China), and all patients provided written informed consent. 


\section{Angiogram and analysis}

Coronary angiography was performed from the radial approach after intracoronary injection of nitroglycerin (100-200 $\mu \mathrm{g})$. Patients were pretreated with aspirin (300 mg) and clopidogrel (300 $\mathrm{mg}$ ) at least $2 \mathrm{~h}$ prior to the index procedure, followed by aspirin $100 \mathrm{mg}$ daily and clopidogrel $75 \mathrm{mg}$ daily for a minimum of 1 year. A non-culprit lesion had to be at least $10 \mathrm{~mm}$ away from the culprit lesion. Any plaque with luminal narrowing $>30 \%$ on an angiogram was included in the analysis. Coronary angiograms were analysed at an independent core laboratory using a quantitative coronary angiogram analysis (QCA) program (Quantcor OCA 5.0, Pie Medical Imaging BV. Maastricht, The Netherlands) by two angiographers who were blinded to clinical information. Minimal luminal diameter (MLD, mm) was defined as the smallest lumen diameter in the segment of lesion; reference vessel diameter (RVD, $\mathrm{mm}$ ) was defined as the averaged diameter of the proximal and distal coronary segments without obvious narrowing within $10 \mathrm{~mm}$ from the lesion edge; diameter stenosis (DS,\%) was defined as (RVD-MLD)/RVD $\times 100 \%$; lesion length ( $\mathrm{mm}$ ) was defined as the length of the stenotic segment calculated from the two points (proximal and distal) where the coronary margins change direction and create shoulders between the angiographically normal segments and the diseased segments.

\section{OCT imaging and analysis}

OCT imaging was performed with a 0.016 -inch OCT system (ImageWire, LightLab Imaging, Westford, Massachusetts, USA). The catheter was advanced beyond the distal end of the plaque through a 3-F occlusion balloon catheter. In order to remove red blood cells from the field of view, an occlusion balloon was inflated to $0.5-0.7 \mathrm{~atm}$ at the proximal site of the plaque and lactate Ringer's solution was infused into the coronary artery from the distal tip of the occlusion balloon catheter at $0.5 \mathrm{ml} / \mathrm{s}$ by an injector. The vessel was imaged with an automatic pullback device at $3 \mathrm{~mm} / \mathrm{s}$.

\section{OCT data collection and analysis}

OCT image analysis was performed at an independent core laboratory at the Massachusetts General Hospital. Two independent reviewers blinded to the clinical information performed the assessment of all OCT images according to the criteria of the Clinical Expert Consensus Document on OCT. ${ }^{8}$ Any discordance between the two independent reviewers was resolved by consensus.

A NV was defined as a small black hole within a plaque with a diameter of $50-300 \mu \mathrm{m}$ that was present on at least three consecutive frames in pull-back images (figures 1 and 2). ${ }^{9-11}$ The presence of plaque disruption or thrombus was also noted. Plaque disruption was defined as a fibrous cap discontinuity with cavity formation. Thrombus was defined as an irregular mass protruding into the lumen that had a measured dimension $\geq 250 \mu \mathrm{m}$. Fibrous cap thickness covering lipid core was measured three times at its thinnest location and the mean value of the three measurements was used for subsequent analysis. For ruptured plaque, the residual fibrous cap was identified as a flap between the lumen and the cavity of the plaque, and its thickness was measured three times at its thinnest part. Plaque lipid content was semi-quantified according to the maximal value of involved arc on the cross-sectional OCT image. ${ }^{12}$ When a plaque included a lipid arc $>90^{\circ}$, it was considered lipid-rich. Thin cap fibroatheroma (TCFA) was defined as lipid-rich plaque with fibrous cap thickness $\leq 65 \mu \mathrm{m}$. The longitudinal length of lipid core was determined by the numbers of OCT images at $1 \mathrm{~mm}$ intervals. Calcification was also recorded when an area with low backscatter and a sharp border was identified inside a plaque.

\section{IVUS imaging and analysis}

The methods of IVUS imaging and analysis are provided in the online supplementary materials.

\section{Statistical analysis}

Plaques with and without NV were compared in each group. All statistical analysis was performed by an independent statistician at a core laboratory. Categorical variables were expressed as numbers or frequencies of occurrence. All continuous variables were tested for normal distribution by the non-parametric onesample Kolmogorov-Smirnov test and normal probability (Q-Q) plots. Continuous variables were expressed as means \pm SD for normally distributed variables and as medians $(25-75$ th percentiles) for non-normally distributed variables. Betweengroup differences in baseline patient characteristics were tested
Figure 1 Representative optical coherence tomography images of coronary plaque with neovascularisation (red arrows). The longitudinal pull back image $(A)$ of the vessel with the locations of three cross sectional images ( $B, C$ and $D)$. Three consecutive images show that microvessel connects adventitia and the plaque close to the lumen.
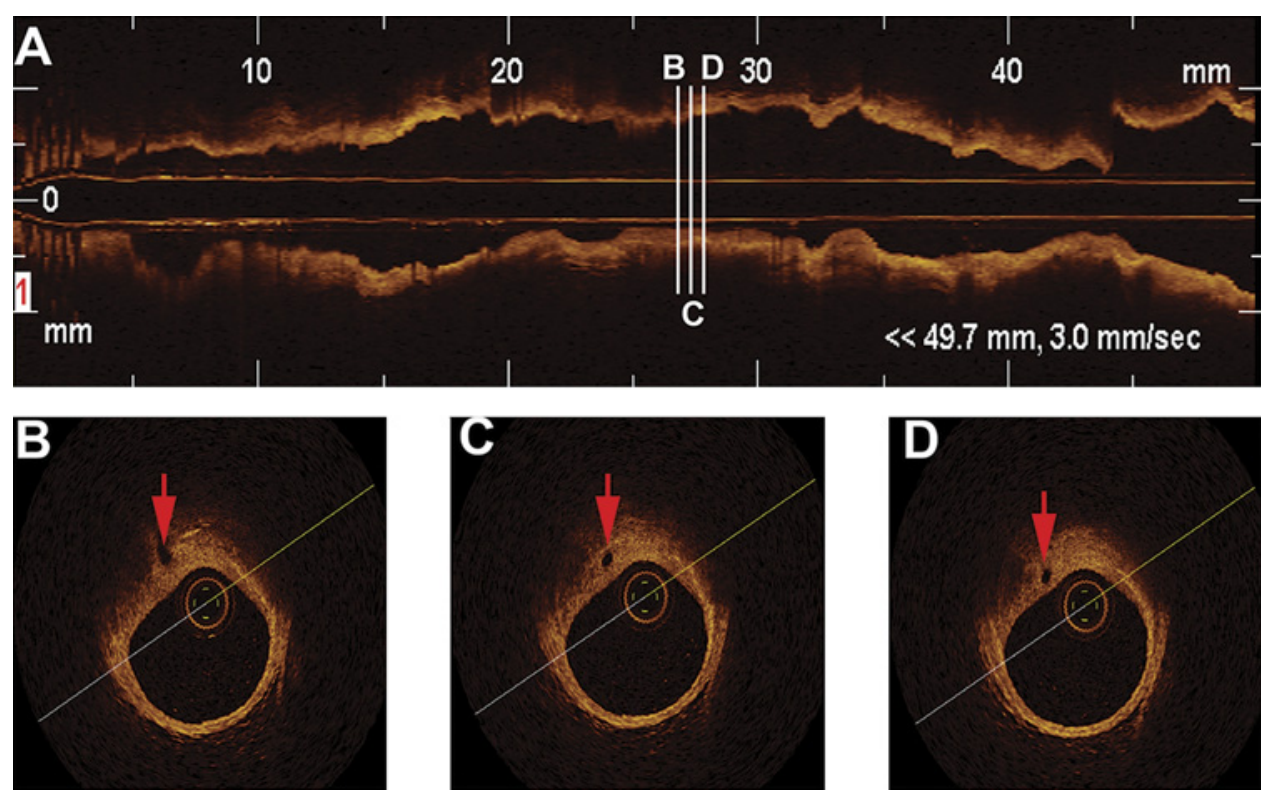
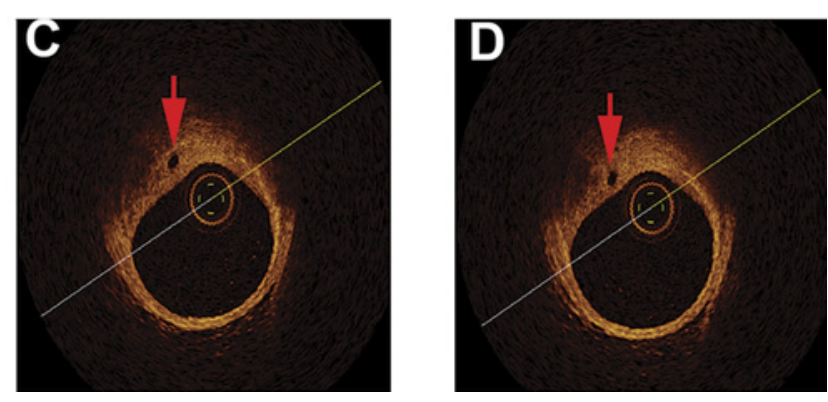
Figure 2 Representative optical coherence tomography images of ruptured plaque with neovascularisation (red arrows). The longitudinal pull back image $(A)$ of the vessel with the locations of five cross sectional images (B-G). Neovascularisation (red arrows) were located at the shoulder region of plaque $(B, C$ and $D)$. Plaque rupture (white arrows) was visualised at 7o'clock position (E, F and G). Minimum lumen area site $(\mathrm{G})$.
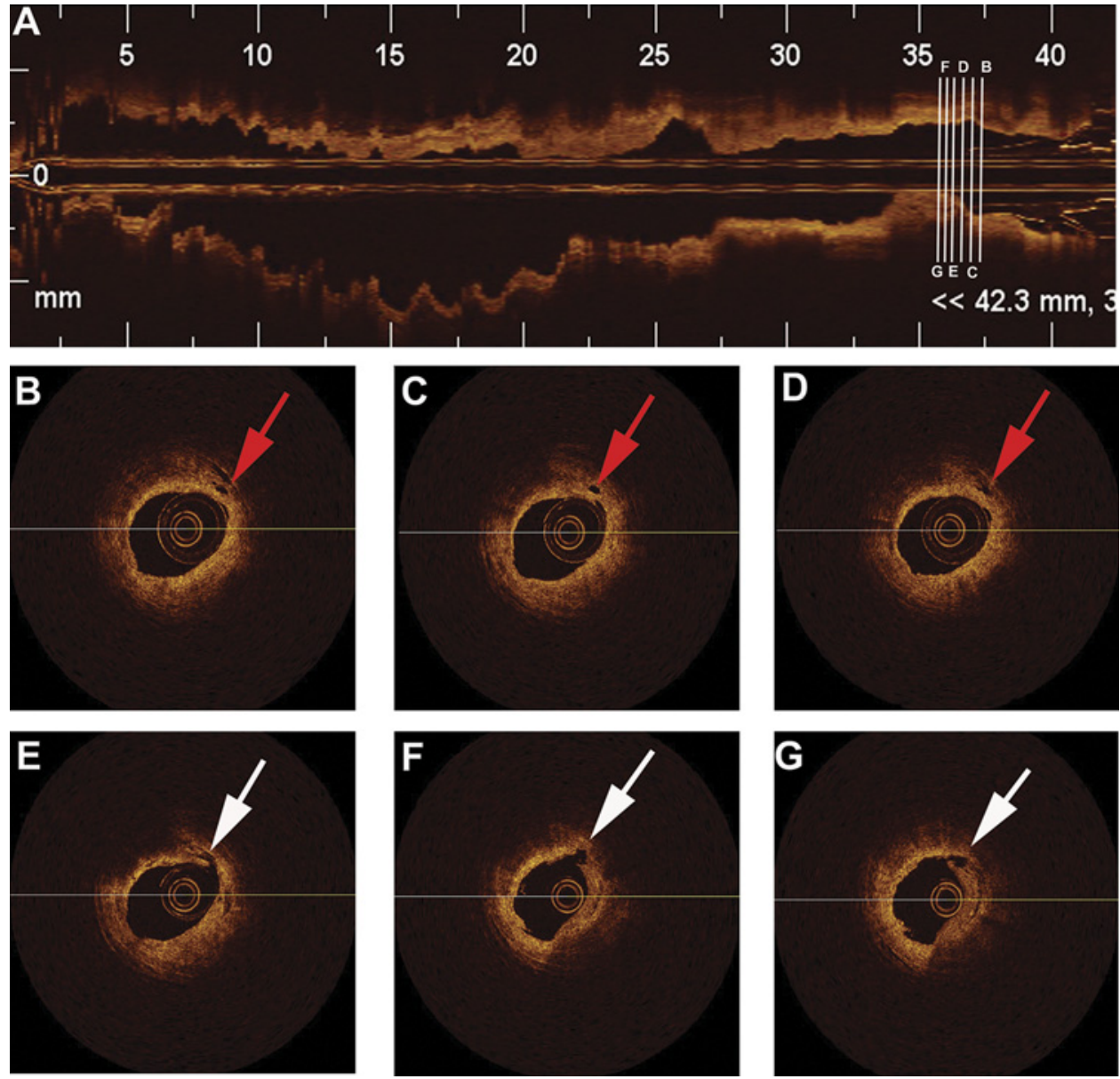

using $\chi^{2}$-square test or Fisher's exact test for binary variables and using independent samples t-test. For the between-group comparisons of the plaque characteristics it was necessary to take into account the multiple plaques in culprit- and nonculprit lesions within a unique patient, and the generalised estimating equations approach was applied. For continuous plaque characteristics measures, the identity mean link function with Gaussian variance was used; and for binary measures, the logit mean function with binomial variance was used. All analysis was performed using SAS V.9.1.3. A $p$ value $<0.05$ was considered statistically significant.

\section{RESULTS}

Baseline clinical characteristics and laboratory data

There was no significant difference in the baseline characteristics between the groups with or without NV except for the higher frequency of smoking in the UAP group in non-culprit lesions with NV (67\% vs $50 \%, \mathrm{p}=0.02)$ (table 1$)$.

\section{Angiographic plaque characteristics}

The distribution of plaques among coronary arteries was similar: the left anterior descending artery was the most frequent (49\%), followed by the right coronary artery (34\%) and the circumflex

Table 1 Patient characteristics

\begin{tabular}{|c|c|c|c|c|c|c|c|c|c|}
\hline \multirow[b]{2}{*}{ Variable } & \multicolumn{2}{|c|}{ Culprit lesions in UAP } & \multirow[b]{2}{*}{ p Value } & \multicolumn{2}{|c|}{ Non-culprit lesions in UAP } & \multirow[b]{2}{*}{ p Value } & \multicolumn{2}{|c|}{ Lesions in SAP } & \multirow[b]{2}{*}{ p Value } \\
\hline & $N V(n=32)$ & No NV $(n=60)$ & & $N V(n=70)$ & No NV $(n=133)$ & & $N V(n=17)$ & No NV $(n=44)$ & \\
\hline Men, n (\%) & $25(78)$ & $42(70)$ & 0.40 & $52(74)$ & $89(67)$ & 0.28 & $10(59)$ & $29(66)$ & 0.61 \\
\hline Age, years & $57 \pm 11$ & $58 \pm 8$ & 0.66 & $59 \pm 8$ & $58 \pm 9$ & 0.39 & $54 \pm 7$ & $55 \pm 8$ & 0.44 \\
\hline Smoker, n (\%) & $18(56)$ & $30(50)$ & 0.57 & 47 (67) & $66(50)$ & 0.02 & $8(47)$ & $16(36)$ & 0.44 \\
\hline Hypertension, n (\%) & $19(59)$ & $31(52)$ & 0.48 & $43(61)$ & $70(53)$ & 0.23 & $12(71)$ & $25(57)$ & 0.32 \\
\hline Dyslipidaemia, n (\%) & $17(53)$ & $35(58)$ & 0.63 & $32(45)$ & $69(52)$ & 0.40 & $9(53)$ & $23(52)$ & 0.96 \\
\hline Diabetes, n (\%) & $9(28)$ & $20(33)$ & 0.61 & $34(49)$ & $54(41)$ & 0.28 & $7(41)$ & $14(32)$ & 0.49 \\
\hline Family history & $7(22)$ & $19(32)$ & 0.32 & $20(29)$ & $35(26)$ & 0.73 & $5(29)$ & $15(34)$ & 0.73 \\
\hline \multicolumn{10}{|l|}{ Medications } \\
\hline Aspirin, n (\%) & 31 (97) & $59(98)$ & 1.00 & $68(97)$ & $131(99)$ & 0.61 & $16(94)$ & $37(84)$ & 0.54 \\
\hline Clopidogrel, n (\%) & $28(88)$ & $53(88)$ & 1.00 & $65(93)$ & $125(94)$ & 0.99 & $12(71)$ & $35(80)$ & 0.68 \\
\hline Statin, n (\%) & 31 (97) & $53(88)$ & 0.32 & $60(86)$ & $113(85)$ & 0.87 & $15(88)$ & $42(96)$ & 0.31 \\
\hline ACEI or ARB, $\mathrm{n}(\%)$ & $18(56)$ & $28(47)$ & 0.38 & $36(51)$ & $62(47)$ & 0.51 & $9(53 \%)$ & $20(46)$ & 0.60 \\
\hline
\end{tabular}

Data are presented as mean \pm SD, $n(\%)$.

ACEl, ACE inhibitor; ARB, angiotensin II receptor blockers; NV, neovascularisation; SAP, stable angina pectoris; UAP, unstable angina pectoris; SAP, stable angina pectoris. 
Table 2 Quantitative coronary angiography findings

\begin{tabular}{|c|c|c|c|c|c|c|c|c|c|}
\hline \multirow[b]{2}{*}{ Variable } & \multicolumn{2}{|c|}{ Culprit lesions in UAP } & \multirow[b]{2}{*}{ p Value } & \multicolumn{2}{|c|}{ Non-culprit lesions in UAP } & \multirow[b]{2}{*}{ p Value } & \multicolumn{2}{|c|}{ Lesions in SAP } & \multirow[b]{2}{*}{ p Value } \\
\hline & $\mathrm{NV}(\mathrm{n}=32)$ & No NV $(n=60)$ & & $\mathrm{NV}(n=70)$ & No NV $(n=133)$ & & $\mathrm{NV}(\mathrm{n}=17)$ & No NV $(n=44)$ & \\
\hline \multicolumn{10}{|l|}{ Artery, n (\%) } \\
\hline LCX & $8(25)$ & $7(12)$ & & $13(19)$ & $23(17)$ & & $3(18)$ & $8(18)$ & \\
\hline RCA & $10(31)$ & $22(37)$ & & $20(29)$ & $54(41)$ & & $5(29)$ & $10(23)$ & \\
\hline RVD, mm & $2.86 \pm 0.61$ & $3.01 \pm 0.68$ & 0.32 & $2.83 \pm 0.68$ & $2.96 \pm 0.69$ & 0.22 & $2.83 \pm 0.51$ & $3.16 \pm 0.59$ & 0.049 \\
\hline MLD, mm & $0.99 \pm 0.34$ & $1.01 \pm 0.40$ & 0.74 & $1.76 \pm 0.50$ & $1.80 \pm 0.52$ & 0.63 & $1.65 \pm 0.58$ & $1.53 \pm 0.64$ & 0.52 \\
\hline Lesion length, mm & $11.2 \pm 3.9$ & $13.2 \pm 8.1$ & 0.20 & $11.3 \pm 8.3$ & $10.4 \pm 6.6$ & 0.40 & $10.2 \pm 4.1$ & $11.3 \pm 5.4$ & 0.45 \\
\hline
\end{tabular}

Data are presented as mean $\pm \mathrm{SD}, \mathrm{n}(\%)$.

DS, diameter stenosis; LAD, left anterior descending artery; LCX, left circumflex arter; MLD, minimum lumen diameter; NV, neovascularisation; RCA, right coronary artery; RVD, reference vesse diameter; SAP, stable angina pectoris; UAP, unstable angina pectoris.

artery (17\%). Patients with UAP had an average of 3.2 plaques, while patients with SAP had an average of 2.4 plaques $(p=0.018)$. Compared with SAP lesions without NV, SAP lesions with NV had a bigger reference vessel diameter $(\mathrm{p}=0.049)$ (table 2$)$.

\section{IVUS findings}

IVUS was performed only in a subset of 35 UAP patients. Plaque burden was significantly larger in UAP culprit lesions (or vessels) with NV than in those without NV $(79.8 \pm 7.9$ vs $72.8 \pm 10.7$, $\mathrm{p}=0.024)$. Plaque burden was marginally bigger in non-culprit lesions with NV than in those without NV $(p=0.051)$. There was no difference in remodelling index (see online supplementary table 1).

\section{OCT findings}

The total length of the coronary arteries examined by OCT was $76 \pm 21 \mathrm{~mm}$ in the left anterior descending artery, $68 \pm 13 \mathrm{~mm}$ in the left circumflex artery, and $72 \pm 17 \mathrm{~mm}$ in the right coronary artery. The incidence of intraplaque NV was $35 \%$ in UAP culprit lesions, $34 \%$ in UAP non-culprit lesions, and $28 \%$ in SAP lesions $(\mathrm{p}=0.60)($ table 3$)$.

Among UAP culprit lesions, plaques with NV had significantly higher incidence of TCFA compared with those without NV ( $81 \%$ vs $47 \%, p=0.002)$. In addition, fibrous cap was thinner $(56 \pm 20 \mu \mathrm{m}$ vs $75 \pm 30 \mu \mathrm{m}, \mathrm{p}<0.001)$, lipid arc was greater $\left(254 \pm 66^{\circ}\right.$ vs $\left.222 \pm 65^{\circ}, p=0.024\right)$, and lipid core length was longer $(13 \pm 5 \mathrm{~mm}$ vs $10 \pm 6 \mathrm{~mm}, \mathrm{p}=0.007)$. In the non-culprit lesions of UAP patients and lesions of SAP patients, no difference in plaque characteristics was observed between those with and without NV. Figure 2 shows representative OCT images of a ruptured plaque with $\mathrm{NV}$.

\section{DISCUSSION}

Our study demonstrated that vulnerable plaque characteristics, including thinner fibrous cap, greater lipid arc, longer lipid core length, and TCFA, were seen more frequently in UAP culprit lesions with NV when compared with those without $\mathrm{NV}$, but not in the non-culprit lesions of UAP and in the lesions of SAP.

In this study, we observed NV in $35 \%$ of the culprit lesions in UAP patients. This finding is consistent with the previous report by Kitabata et al, ${ }^{9}$ where NV was found in $38 \%$ of the culprit lesions in UAP patients. NV was observed in 34\% of non-culprit lesions and in $28 \%$ of the lesions in SAP patients. The difference was not significant between the three groups. Our findings, which associate NV with the presence of TCFA, thinner fibrous cap, and larger plaque burden, are also in good agreement with the previous report. ${ }^{9}$ The results obtained from the study by Fujii $\mathrm{K}$ et $a l^{13}$ have shown that ruptured plaques in culprit lesions of patients with acute coronary syndromes have greater plaque burdens. In this study, IVUS analyses have demonstrated that plaque burden was significantly bigger in culprit lesions with NV. Notably, our results demonstrate that only the NV in the culprit lesions was associated with the plaque vulnerability.

Previous studies have demonstrated that NV was larger and more irregular in symptomatic carotid atherosclerotic plaque than that in asymptomatic plaque. ${ }^{67}$ Symptomatic patients had more intense contrast agent enhancement, a quantification index of NV, in the plaque than asymptomatic patients. ${ }^{14} 15$ The irregular NV from symptomatic plaques was more aberrant, highly immature, and leaky, similar to those found in cancers. ${ }^{6}$ We attempted to find NV features specific for unstable lesions. However, due to limitations of OCT technology we were not able to find OCT characteristics for unstable NV. Instead, our study has shown that the characteristics of plaque instability, such as thinner fibrous cap, greater lipid arc, longer lipid core length, and TCFA, were seen more frequently in UAP culprit lesions with NV, but not in non-culprit lesions. Our results have corroborated the concept that the role of $\mathrm{NV}$ in active and inactive plaques differs and depends on the stage of disease. In

Table 3 Optical coherence tomography findings

\begin{tabular}{|c|c|c|c|c|c|c|c|c|c|}
\hline \multirow[b]{2}{*}{ Variable } & \multicolumn{2}{|c|}{ Culprit lesions in UAP } & \multirow[b]{2}{*}{ p Value } & \multicolumn{2}{|c|}{ Non-culprit lesions in UAP } & \multirow[b]{2}{*}{ p Value } & \multicolumn{2}{|c|}{ Lesions with SAP } & \multirow[b]{2}{*}{ p Value } \\
\hline & $\mathrm{NV}(\mathrm{n}=32)$ & No NV $(n=60)$ & & $\mathrm{NV}(n=70)$ & No NV $(n=133)$ & & $\overline{N V}(n=17)$ & No NV $(n=44)$ & \\
\hline TCFA, n (\%) & $26(81)$ & $28(47)$ & 0.002 & $27(39)$ & $56(42)$ & 0.62 & $7(41)$ & $19(43)$ & 0.90 \\
\hline Fibrous cap thickness, $\mu \mathrm{m}$ & $56 \pm 20$ & $75 \pm 30$ & $<0.001$ & $90 \pm 44$ & $97 \pm 61$ & 0.36 & $83 \pm 36$ & $93 \pm 61$ & 0.48 \\
\hline Lipid core length, mm & $13 \pm 5$ & $10 \pm 6$ & 0.007 & $9 \pm 6$ & $8 \pm 4$ & 0.11 & $10 \pm 4$ & $9 \pm 6$ & 0.51 \\
\hline Calcification, n (\%) & $11(34)$ & $22(37)$ & 0.83 & $29(41)$ & $41(31)$ & 0.24 & $7(41)$ & $13(30)$ & 0.50 \\
\hline Disruption, n (\%) & $18(56)$ & $31(52)$ & 0.67 & $6(9)$ & $8(6)$ & 0.51 & $1(6)$ & $4(9)$ & 0.76 \\
\hline
\end{tabular}

Data are presented as mean $\pm \mathrm{SD}, \mathrm{n}(\%)$.

NV, neovascularisation; SAP, stable angina pectoris; TCFA, thin cap fibroatheroma; UAP, unstable angina pectoris. 
contrast to myocardium ischaemia, in which NV is beneficial, $\mathrm{NV}$ within atherosclerotic plaques is a double-edged sword depending on the stage of the disease. ${ }^{16}$ At the early stage, the neoangiogenesis functions as a conduit for the supply of nutrients and oxygen and serves to protect the vessel wall from ischaemic damage. With imbalance between antiangiogenic and proangiogenic factors, ${ }^{17}$ the NV becomes more immature and leaky, promoting the conversion of a stable plaque to an unstable phenotype. ${ }^{16}$ One OCT study has demonstrated that microchannel was a predictor of subsequent plaque progression in patients with coronary heart disease. ${ }^{11}$ Due to lack of mural pericytes, smooth muscle cells, and complete endothelial cell junctions, ${ }^{5}$ immature $\mathrm{NV}$ is prone to rupture and to induce haemorrhages within atherosclerotic lesions. This is a stimulus for the progression of atherosclerosis by increasing necrotic core volume and triggering local inflammation. ${ }^{18}$ Autologous erythrocytes delivered into experimental plaques promote the formation of large necrotic core and macrophage infiltration. ${ }^{18}$ Active inflammatory cells secrete more proangiogenic factors. Some studies have demonstrated that both macrophage infiltration and intraplaque NV are usually seen in the 'shoulder' regions of plaques, ${ }^{19} 20$ which are considered to be prone to rupture. Matrix-degrading enzymes produced by inflammatory cells may damage intraplaque angiogenesis, weaken fibrous cap, and destabilise the plaque. ${ }^{21}$ One OCT study has demonstrated that inflammatory mediators including highly sensitive $C$ reactive protein, IL-18 and tumour necrosis factor $\alpha$ have significant negative relationship with the thickness of fibrous cap. ${ }^{22}$ The emerging data suggest that the inflammatory process is indeed a precursor of the clinical event. ${ }^{20} 22$ In addition, intraplaque NV permits cholesterol-enriched erythrocyte cells into the plaque, ${ }^{3} 1618$ producing abundant cholesterol material for crystallisation of cholesterol. Crystallisation of supersaturated cholesterol in atherosclerotic plaques can also induce fibrous cap disruption. ${ }^{23}$ An autopsy study has reported that the presence of cholesterol crystal is an independent predictor of thrombus and clinical manifestation. ${ }^{24}$ Cholesterol crystallisation rapidly changes in the plaque spatial configuration, pierces the arterial plaque and intima, ${ }^{25}$ and possibly perforates intraplaque $\mathrm{NV}$. Thus, it has been postulated that the intraplaque NV, active inflammation, and cholesterol crystals interact synergistically to convert stable plaque to unstable, resulting in plaque rupture. ${ }^{16} 26$ Atherosclerosis is a systemic disease and because patients usually harbour multiple plaques which are at different stage, we need to understand in which lesions angiogenesis is beneficial or harmful. ${ }^{1627}$ This will effectively guide us for target therapy. ${ }^{5}$ Some side effects regarding the systemic use of angiogenesis inhibitors are that they can suppress collateral development and aggravate organ ischaemia, for example, myocardial ischaemia, and the risk for arterial thromboembolic events. ${ }^{28}$

Current coronary imaging modalities, such as coronary angiography, IVUS and multi-slice CT, lack sufficient resolution to detect plaque angiogenesis. Molecular imaging with the use of intravenous agents (eg, av $\beta 3$-targeted nanoparticle MRI contrast agents ${ }^{29}$ ) offers new ways to detect plaque angiogenesis in the experimental atherosclerotic model, but further work is needed before these techniques can be tested in humans. Some studies have reported that IVUS-enhancement imaging with microbubble contrast agents can image ectopic NV in coronary atherosclerotic plaques in vivo ${ }^{30}$; however, additional studies are required to show the feasibility of this method in a routine clinical setting. OCT is an emerging high-resolution intravascular imaging modality with excellent resolution. OCT has displayed the ability to clearly visualise NV within atheroscle- rotic plaques and to dynamically evaluate the effects of various therapeutic strategies on plaque stability in vivo.

Several limitations in this study should be mentioned. Although we studied consecutive patients to minimise selection bias, this was a retrospective study with inherent potential bias. The penetration of OCT is limited, particularly in lipid-rich plaque and in the presence of red thrombus. Due to this pitfall, the microvessels at the base of plaque might have been underestimated. In addition, with two dimensional OCT images it is often difficult to locate NV. Therefore, we did not attempt to measure the density. Detection of intraplaque haemorrhage and necrotic core by OCT has not been validated. Therefore, these changes were not reported in our study. Patients with acute myocardial infarction were excluded because of the concern that residual mural thrombus might interfere with accurate evaluation of NV. Finally, IVUS was used only in a subset of patients.

\section{CONCLUSION}

In conclusion, vulnerable plaque characteristics such as thin fibrous cap, greater lipid arc, longer lipid core length, and TCFA were seen more frequently in UAP culprit lesions with NV, which indicate possible role of $\mathrm{NV}$ in destabilisation of a plaque.

Acknowledgements The authors thank Yun Xiao and SongYu Tian for their help in editing assistance; and the core laboratory of Massachusetts General Hospital OCT Registry for their efforts to ensure the accuracy of the data submitted.

Contributors JT, BY and IKJ participated in the conception and design, carried out data collection and analyses, and wrote the main parts of the manuscript. JH, LX and SZ participated in the conception and design and made substantial contributions to the manuscript. SJK, TY, KK and HL made substantial contributions to the acquisition of data and revising the manuscript. All authors have given their final approval of the version to be published.

Funding This study was supported by grant $81171430 / \mathrm{H} 1816$ from the National Natural Science Foundation of China, and grant GC10C305-3 from the Science and Technology Key Project of Heilongjiang Province, China.

Competing interests $\mathrm{SZ}$ is an employee of LightLab Imaging/St. Jude Medical. IKJ received a research grant for the MGH Registry from LightLab Imaging/St. Jude Medical. JT, BY, JH, LX, SJK, TY, KK and HL report no conflicts.

Patient consent Obtained.

Ethics approval This study was approved by the ethics committee of the 2nd Affiliated Hospital of Harbin Medical University (Harbin, China).

Provenance and peer review Not commissioned; externally peer reviewed.

\section{REFERENCES}

1. Kumamoto M, Nakashima $Y$, Sueishi K. Intimal neovascularization in human coronary atherosclerosis: its origin and pathophysiological significance. Hum Pathol 1995;26:450-6.

2. Fleiner $\mathbf{M}$, Kummer $\mathbf{M}$, Mirlacher $\mathbf{M}$, et al. Arterial neovascularization and inflammation in vulnerable patients: early and late signs of symptomatic atherosclerosis. Circulation 2004;110:2843-50.

3. Moreno PR, Purushothaman KR, Fuster V, et al. Plaque neovascularization is increased in ruptured atherosclerotic lesions of human aorta: implications for plaque vulnerability. Circulation 2004;110:2032-8.

4. Sluimer JC, Kolodgie FD, Bijnens AP, et al. Thin-walled microvessels in human coronary atherosclerotic plaques show incomplete endothelial junctions relevance of compromised structural integrity for intraplaque microvascular leakage. J Am Coll Cardiol 2009:53:1517-27.

5. Zachary I, Morgan RD. Therapeutic angiogenesis for cardiovascular disease biological context, challenges, prospects. Heart 2011:97:181-9.

6. Dunmore BJ, McCarthy MJ, Naylor AR, et al. Carotid plaque instability and ischemic symptoms are linked to immaturity of microvessels within plaques. J Vasc Surg 2007;45:155-9.

7. McCarthy MJ, Loftus IM, Thompson MM, et al. Angiogenesis and the atherosclerotic carotid plaque: an association between symptomatology and plaque morphology. J Vasc Surg 1999:30:261-8.

8. Prati F, Regar E, Mintz GS, et al. Expert review document on methodology, terminology, and clinical applications of optical coherence tomography: physical principles, methodology of image acquisition, and clinical application for assessment of coronary arteries and atherosclerosis. Eur Heart $J$ 2010;31:401-15. 
9. Kitabata H, Tanaka A, Kubo T, et al. Relation of microchannel structure identified by optical coherence tomography to plaque vulnerability in patients with coronary artery disease. Am J Cardiol 2010;105:1673-8.

10. Vorpahl M, Nakano M, Virmani R. Small black holes in optical frequency domain imaging matches intravascular neoangiogenesis formation in histology. Eur Heart $J$ 2010;31:1889.

11. Uemura S, Ishigami Kl, Soeda T, et al. Thin-cap fibroatheroma and microchannel findings in optical coherence tomography correlate with subsequent progression of coronary atheromatous plaques. Eur Heart $J$ 2012;33:78-85.

12. Tanaka A, Imanishi $\mathrm{T}$, Kitabata $\mathrm{H}$, et al. Lipid-rich plaque and myocardial perfusion after successful stenting in patients with non-ST-segment elevation acute coronary syndrome: an optical coherence tomography study. Eur Heart J 2009;30:1348-55.

13. Fujii K, Kobayashi Y, Mintz GS, et al. Intravascular ultrasound assessment of ulcerated ruptured plaques: a comparison of culprit and nonculprit lesions of patients with acute coronary syndromes and lesions in patients without acute coronary syndromes. Circulation 2003;108:2473-8.

14. Xiong L, Deng YB, Zhu Y, et al. Correlation of carotid plaque neovascularization detected by using contrast-enhanced US with clinical symptoms. Radiology 2009;251:583-9.

15. Staub D. Patel MB, Tibrewala $A$, et al. Vasa vasorum and plaque neovascularization on contrast-enhanced carotid ultrasound imaging correlates with cardiovascular disease and past cardiovascular events. Stroke 2010;41:41-7.

16. Virmani R, Kolodgie FD, Burke AP, et al. Atherosclerotic plaque progression and vulnerability to rupture: angiogenesis as a source of intraplaque hemorrhage. Arterioscler Thromb Vasc Biol 2005;25:2054-61.

17. Jain RK, Finn AV, Kolodgie FD, et al. Antiangiogenic therapy for normalization of atherosclerotic plaque vasculature: a potential strategy for plaque stabilization. Nat Clin Pract Cardiovasc Med 2007:4:491-502.

18. Kolodgie FD, Gold HK, Burke AP, et al. Intraplaque hemorrhage and progression of coronary atheroma. N Engl J Med 2003;349:2316-25.
19. Khurana R, Simons M, Martin JF, et al. Role of angiogenesis in cardiovascular disease: a critical appraisal. Circulation 2005;112:1813-24.

20. Mulvihill NT, Foley JB. Inflammation in acute coronary syndromes. Heart 2002;87:201-4.

21. Newby AC, George SJ, Ismail Y, et al. Vulnerable atherosclerotic plaque metalloproteinases and foam cell phenotypes. Thromb Haemost 2009;101:1006-11.

22. Li OX, Fu 00, Shi SW, et al. Relationship between plasma inflammatory markers and plaque fibrous cap thickness determined by intravascular optical coherence tomography. Heart 2010;96:196-201.

23. Abela GS, Aziz K. Cholesterol crystals cause mechanical damage to biologica membranes: a proposed mechanism of plaque rupture and erosion leading to arteria thrombosis. Clin Cardiol 2005;28:413-20.

24. Abela GS, Aziz K, Vedre A, et al. Effect of cholesterol crystals on plaques and intima in arteries of patients with acute coronary and cerebrovascular syndromes. Am J Cardiol 2009:103:959-68.

25. Abela GS. Cholesterol crystals piercing the arterial plaque and intima trigger local and systemic inflammation. J Clin Lipidol 2010:4:156-64.

26. O'Brien ER, Garvin MR, Dev R, et al. Angiogenesis in human coronary atherosclerotic plaques. Am J Pathol 1994;145:883-94.

27. Ribatti D, Levi-Schaffer F, Kovanen PT. Inflammatory angiogenesis in atherogenesis-a double-edged sword. Ann Med 2008;40:606-21.

28. Choueiri TK, Schutz FA, Je Y, et al. Risk of arterial thromboembolic events with sunitinib and sorafenib: a systematic review and meta-analysis of clinical trials. J Clin Oncol 2010;28:2280-5

29. Winter PM, Morawski AM, Caruthers SD, et al. Molecular imaging of angiogenesis in early-stage atherosclerosis with alpha(v)beta3-integrin-targeted nanoparticles. Circulation 2003;108:2270-4.

30. Gulel 0. Sipahi I, Murattuzcu E, et al. Coronary artery wall enhances with intracoronary injection of echocontrast media during in vivo intravascular ultrasound. Eur J Echocardiogr 2007;8:234-6.

Heart online

Visit Heart online for free editor's choice articles, online archive, email alerts, blogs or to submit your paper. Keep informed and up to date by registering for electronic table of contents at heart.bmj.com. 